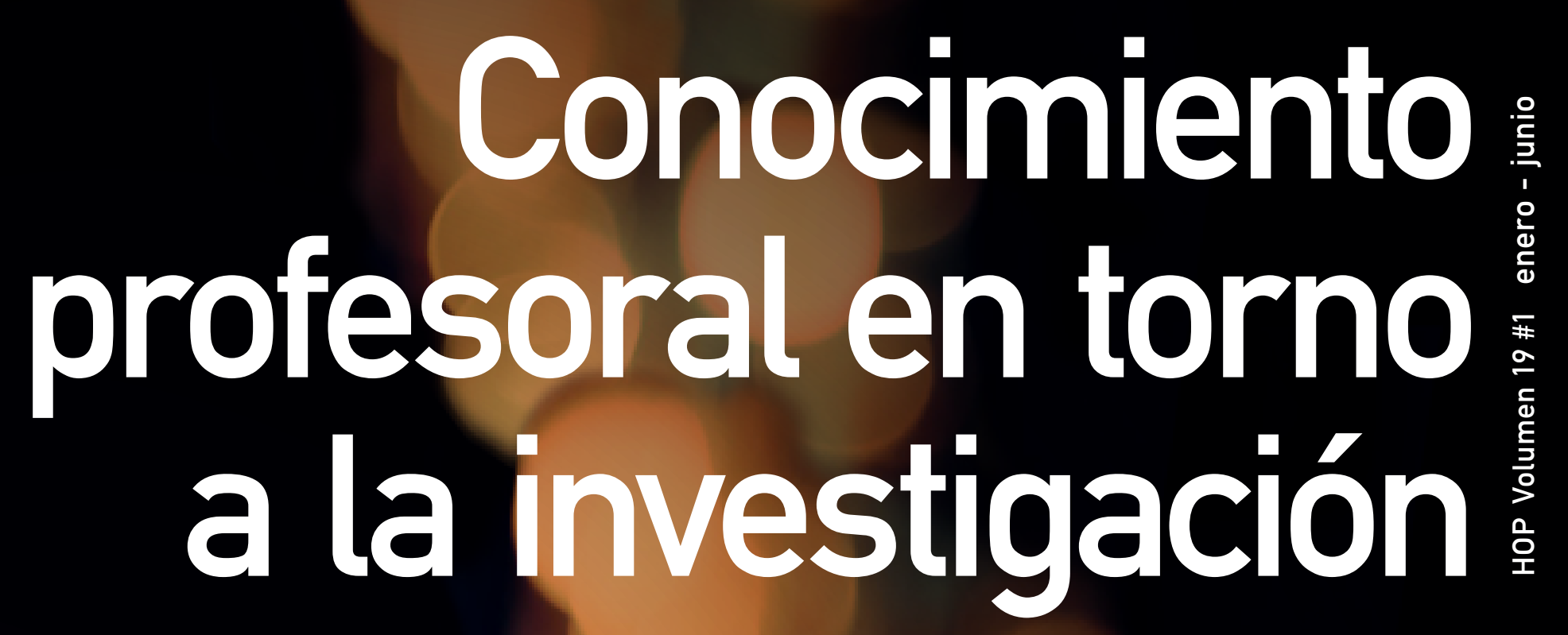

Academic knowledge about research

(c) (i)

(U)

IBEROAMERICANA CORPORACIÓN UNIVERSITARIA

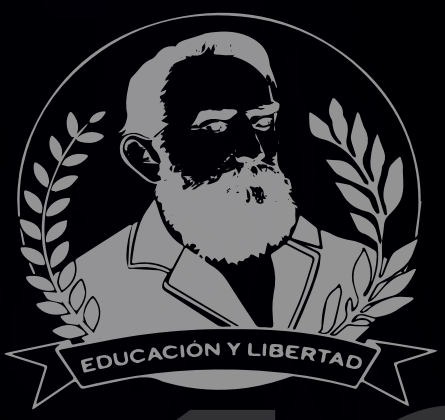

HORIZONTES PEDAGÓGICOS ISSN-I: 0123-8264 | e-ISSN: 2500-705X 


\section{HORIZONTES PEDAGÓGICOS}

0123-8264.hop.19104

Title: $\quad$ Academic knowledge about research

Título: Conocimiento profesoral en torno a la investigación

Alt Title / Título alternativo:

[en]: Academic knowledge about research

[es]: $\quad$ Conocimiento profesoral en torno a la investigación

Author (s) / Autor (es):

Zapata Lesmes, \& Pereira Lentino

Keywords / Palabras Clave:

[en]: $\quad$ knowledge about research; beliefs and conceptions about research; teacher"s thinking; research teaching practices

[es]: $\quad$ conocimiento sobre investigación; creencias y concepciones sobre investigación; pensamiento del profesor; prácticas docentes investigativas

Submited: 2017-06-10

Acepted: 2017-06-15

\section{Resumen}

Este estudio fue realizado en cinco (5) Instituciones de Educación Superior de

Cartagena de Indias, que desarrollan programas de licenciaturas en educación, el caso lo constituyeron siete (7) profesores

universitarios, considerados como las unidades de análisis, quienes hacen parte de los equipos de docentes investigadores en programas de licenciatura en educación, con el propósito de develar su conocimiento sobre investigación, desde sus concepciones y creencias. La metodología se enmarcó en el paradigma histórico hermenéutico de las ciencias sociales, el diseño metodológico fue un estudio de caso único, se utilizaron y cruzaron tres cuestionarios de entrevistas estructuradas, las cuales fueron sometidas a análisis de discurso, del cual emergieron las categorías: ontológica, Yo-Imagen, metacognitiva, Construcción de conocimiento, Inclusiva, tradición cultural, y la potencialidad afectiva. Se concluye que las concepciones sobre investigación son propias del investigador, quien interpreta, investiga y transforma a partir de lo observado, para concebir el fenómeno de forma propia.

Clemencia del Consuelo Zapata Lesmes, MEd Research ID: N-1067-2017

ORCID: $\quad$ 0000-0002-4165-4268

\section{Source I Filiacion:}

Corporación Universitaria Rafael Núñez

BIO:

Magister en Educación, Universidad Santo

Tomás de Colombia

Docente Asociado I

Coordinadora de Investigación en Educación

\section{City I Ciudad:}

Cartagena [co]

e-mail:

clemencia.zapata@curnvirtual.edu.co

\section{Abstract}

Five (5) Higher Education Institutions, in Cartagena de Indias, were the focus of this research, in common, these institutions have bachelor"s programs in education. Seven (7) lectureships teachers constitute the scope of the case, they are the analysis units, and who are part of the research teams in these programs. The purpose is to reveal their knowledge about research, from their conceptions and beliefs on the subject. The methodology was framed in the historical hermeneutic paradigm of the social sciences, based on a single case study. Three questionnaires of structured interviews were used and crossed, which were submitted to discourse analysis, and from which emerged the categories: ontological, I-image, metacognitive, knowledge building, inclusive, cultural tradition, and emotional potential. At the end, the conclusion is peculiar, the conceptions about research from the researchers are open wide to the judgment

of they, where each one is who reviews, interprets, and transforms from the observed, to conceive the phenomenon in its own way.

\section{Yolanda Nohora Pereira Lentino, MEd}

ORCID: $\quad$ 0000-0001-8136-7557

Source I Filiacion:

Corporación Universitaria Rafael Núñez

BIO:

Magister en Educación, Universidad Santo

Tomás de Colombia

Coordinadora de Proyección Social y Práctica

\section{City I Ciudad:}

Cartagena [co]

e-mail:

yolanda.pereira@curnvirtual.edu.co

Citar como:

Zapata Lesmes, C. d., \& Pereira Lentino, Y. N. (2017). Conocimiento profesoral en torno a la investigación. Horizontes Pedagógicos issn-l:0123-8264, 19 (1), 31-38]. Obtenido de: https://revistas.iberoamericana.edu.co/index.php/ rhpedagogicos/article/view/1172 


\section{Conocimiento profesoral en torno a la investigación \\ Academic knowledge about research Clemencia del Consuelo Zapata Lesmes Yolanda Nohora Pereira Lentino}

Esta investigación permitió develar el conocimiento sobre investigación que tiene un grupo de profesores de Licenciaturas en Educación de las Instituciones Educativas de Educación Superior en la ciudad de Cartagena de Indias, en un proceso decomprensión de sus concepcionesy sus creencias. El análisis de discurso realizado se fundamentó en que, tanto las concepciones como las creencias, al ser arraigos del pensamiento humano, son constructos y reproducciones culturales, que en la mente humana se permean y que determinan la forma como quieren ser reconocidos o "percibidos", retomando el término de Cicoruel (1980).

Se tomaron como referentes, postulados de Porland, Rivero García y Martín del Pozo (1998), quienes describen las concepciones desde tres perspectivas, la primera cercana a los planteamientos de Bachelard (1938) y de Claxton (1997), donde "las concepciones son herramientas para interpretar la realidad y para conducirse a través de ella, sin embargo son a la vez, barreras que impiden adoptar perspectivas y cursos de acción libres” (pág. 155), consolidando la mirada constructivista; la segunda perspectiva es llevada al plano de lo sistemático (Claxton, 1997) y de la complejidad (Morin, 1986), donde "las concepciones son consideradas sistemas de ideas en evolución”; la tercera es la perspectiva crítica, planteada desde la dimensión de los valores (Habermas, 1967; Apple, 1979), citados en (Perafán Echeverri, 2002): Pensamiento y conocimiento de los profesores. Debate y perspectivas internacionales, dice que en Colombia muchas personas desde, cierto desconocimiento, se oponen a este tipo de estudios, pero, es necesario abrir el debate sobre las implicaciones del pensamiento y el conocimiento del profesor, y por último Moreno Moreno y Azcarate Giménez (2003), dicen que independiente de, cómo se denomine esta base de conocimientos y significaciones de los sujetos, se constituyen como organizadores implícitos referidos a creencias, significados, conceptos, proposiciones, imágenes mentales y preferencias que influyen tanto en la manera de percibir la realidad como en las prácticas que se implementan. 


\section{Pensamiento del profesor}

En este estudio sobre el pensamiento del profesor, de acuerdo con concepciones significativas planteadas por autores como: Martin del Pozo, Piaget y Vigostky.

Pozo (2006) retoma los planteamientos de Piaget y Vygotsky para explicar la relación de estas dos posturas; según Piaget (1974, citado por (Pozo, 2006), el conocimiento es un proceso de construcción individual, producto de la interacción entre el sujeto con el objeto y el medio, desde esta teoría, el conocimiento es producto de la actividad del sujeto, atendiendo a tres factores: la maduración, la experimentación física y la interacción social. Estos se desarrollan atendiendo a los principios funcionales a los que se refiere Piaget, en donde el individuo al adaptarse debe pasar por situaciones de equilibrio, desequilibrio y acomodación.

En esas posibilidades, factores y situaciones diversas se ven implicadas en procesos de estructuración, reajuste y cambio de sus estructuras mentales, dando lugar a una metodología de construcción de pensamiento individual o particular.

En suma, es fundamental para que el sujeto construya el conocimiento, sus concepciones, sus creencias, su sistema de valores, sus ideas sobre las cosas del mundo que lo rodea; gracias a ello el individuo reajusta y cambia sus estructuras mentales en interacción con su medio, en línea con la posición de Vygotsky (1978), quien le da un valor especial a la interacción del individuo con el medio social para que construya su propio conocimiento, puesto que el conocimiento no solo se construye en la interacción con el medio social, sino que es compartido por los grupos sociales, a través de herramientas culturales compartidas por estos. Para este autor, la cultura y el contexto social en el que se desarrolla una persona son fundamentales en el aprendizaje y en la capacidad para desarrollar las funciones mentales superiores.

En consecuencia, las concepciones, las creenciasy el conocimiento que posee una persona, en este caso el profesor, están mediadas por construcciones cognitivas y meta cognitivas más las interacciones sociales que realiza con otras, en una cultura o en contextos, y que se convierten en construcciones inicialmente personales y que pueden dar lugar a constructos sociales que le permiten explicar los problemas, fenómenos o situaciones del entorno donde interactúa (Gráfico 1).

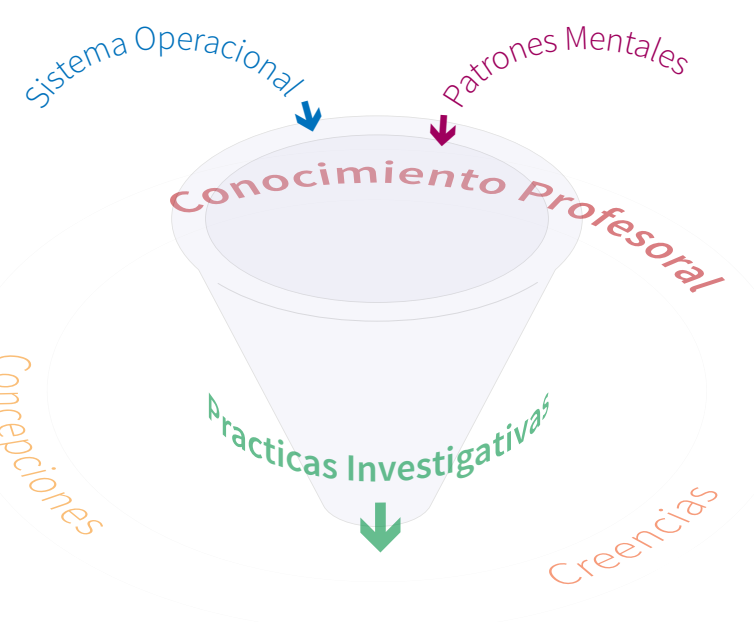

Gráfico 01 Conocimiento profesoral

Fuente: elaboración propia

Los conocimientos y/o saberes profesionales de los profesores desde la perspectiva de Bromme (1988), incluyen "elementos teóricos, reglas empíricas y experiencia práctica” (pág. 23), más otros conocimientos científicos necesarios para el ejercicio de la profesión, en conjunto, estructuran el proceder del profesor y determinan su reflexividad, dada su naturaleza peculiar.
La manera como se asume la investigación en la educación invita a investigar sobre las subjetividades del ser humano que son evidenciadas en su accionar, en su lenguaje, en su manera de interpretar sus realidades e interacciones con el medio. En este trabajo interesa entender las prácticas investigativas de los maestros de las licenciaturas de la ciudad de Cartagena, partiendo de una comprensión de sus concepciones sobre la investigación. El reto que ha asumido la educación actual está en mejorar la calidad del docente en su desempeño como investigador, redefiniendo la concepción investigativa, tomando el modelo de enseñar a pensar a los estudiantes unidos a la investigación.

El conocimiento profesional de los docentes debe formarse en un complejo y prolongado proceso de conocimiento en la acción, de reflexión en y sobre la acción, saber, saber pensary saber investigar, así:

\section{El conocimiento relevante para orientar la práctica del docente en el aula, cuando se propone facilitar el desarrollo de la comprensión en sus alumnos, surge y se genera en la reflexión sobre las características y procesos de su propia práctica, en todas las dimensiones de su amplia aceptación: diseño, desarrollo y evaluación. \\ (Schön, 1987, pág. 119)}

Es evidente que se relaciona con las categorías práctica investigativa, metodológica y planeación de la investigación, que se proponen en este estudio, ya que el docente busca de una u otra forma el desarrollo de la comprensión de sus alumnos de una manera sencilla, utilizando sus métodos aprendidos o construidos en su histórico o dimensiones apropiados que faciliten la reflexión del proceso.

Otro tanto, en esta investigación atañe a las creencias, entendidas hoy en día, como estar en la posesión de una verdad que determina las acciones y forma de actuar del sujeto tanto de forma positiva como negativa. Es decir, incide en el ser humano principalmente en la generación del pensamiento, opciones, aptitudes y actitudes, dada su cultura. "El valor que el docente concede a una creencia, determina el significado que la educación tiene para él”, según Brody y Day (1993, pág. 5); Davies (1993) propone en el campo educativo que determinar las creencias se fundamenta en experiencias personales, sociales -incluye las educativas-, a partir de vivencias en contextos y realidades, así las creencias están sustentadas en esa diversidad de conocimientos adquiridos.

Ampliando lo anterior, King, Shumow y Lietz (2001, citados por Clarke \& Hollingsworth, 2002), indican que cuando el docente trata de elaborar una creencia, se asume para el caso, en el orden de lo investigativo, se:

\section{[... enfrenta en ocasiones a una determinación negativa, debido a las inconsistencias que estos tienen sobre su enseñanza práctica, la pobre adquisición y estabilidad de los conocimientos y la inestabilidad de las habilidades metodológicas". Sin embargo, "la mejora del conocimiento y de sus habilidades ayudan de manera positiva en la creación, asentamiento, elaboración y reformulación de las creencias investigativas.}

(Clarke \& Hollingsworth, 2002, pág. 951)

Se infiere, que la construcción de las creencias investigativas por parte del docente universitario motiva los aprendizajes para superar los obstáculos epistemológicos derivados del proceso de formación y de la misma experiencia en el área. 


\section{Metodología}

Se realizó una investigación cualitativa; se privilegia la hermenéutica, desde los acercamientos de Beuchot (2004), como también desde la perspectiva habermasiana, expuesta en la Lógica de las Ciencias Sociales (Habermas, 1967); se realizó la identificación, análisis e interpretación de las creencias y las concepciones sobre investigación, subyacentes en el pensamiento de siete profesores investigadores de las únicas instituciones de educación superior que en Cartagena de Indias desarrollan programas de licenciaturas en educación.

El diseño metodológico fue un estudio de caso (Yin, 1994), de carácter crítico y único; fue elegido por la particularidad de los sujetos y del objeto del mismo de estudio; de igual forma, se consideró el aporte de Merrian (1997, citado por Álvarez Álvarez \& San Fabián Maroto, 2012), frente a la utilidad de este diseño para estudiar problemas prácticos en situaciones determinadas.

Sevaloró parala inclusión de lossujetos, su formacióny experiencia sobre investigación; luego se recolecto la información, utilizando tres versiones de entrevista, con tres cuestionarios estructurados, ajustados a la formulación de preguntas orientadores de Patton (1990); los cuestionarios a su vez, fueron: el primero adaptado de entrevistas realizadas por Marieta Quintero Mejía y Alexander Ruíz Silva, en su estudio nacional a grupos de investigación, base para su libro: ¿qué significa investigar en Colombia? (2004), con categorías y preguntas propias; en el segundo, se adoptaron cuatro categorías del modelo para salud sana de Becker y Maiman (1975), con preguntas propias; y el tercero se adaptó de un trabajo de investigación realizado por Ayala Rengifo y Martín Cardinal (2010). Al aplicarlas se utilizó el consentimiento y asentimiento informado, avalado previamente por el comité de ética de la Corporación Universitaria Rafael Núñez.

En la tercera fase de Interpretación de resultados, fueron transcritas las entrevistas para identificary categorizar las concepciones y las creencias del profesor sobre investigación, se realizaron dos niveles de análisis y de interpretación: primero, se identificaron proposiciones con carga interpretativa e iniciar el análisis triangular confrontando con el referente teórico, para relacionar los datos obtenidos con las proposiciones o hipótesis definidas como lo indica Yin (1994), en: the logic linking the data to the propositions; luego, las unidades de análisis identificadas fueron interpretadas, en un acercamiento a los criterios de la técnica de análisis de discurso, the criteria for interpreting the findings, también propuesto por Yin (1994), para el estudio de caso.

El análisis discursivo se interesó en los sentidos de los discursos, en las argumentaciones de los profesores, donde evidenciaron formas de producción y de circulación de significación social y cultural, en un intento por posibilitar el reconocimiento de formas de exclusión, de dominación y/o resistencia, formas estereotipadas, etc., como lo anuncia van Dijk. (2001)

\section{Resultados}

Los profesores de las licenciaturas en educación de la ciudad de Cartagena de Indias participantes en este estudio, se han actualizado en seminarios y cursos en el área al interior de la Instituciones de Educación Superior donde laboran, en calidad de asistentes, cursantes y cualificadores y contaban con formación así:
Tabla 1 Sujetos de Investigación

\begin{tabular}{|c|c|}
\hline Código & Descripción del Sujeto \\
\hline KC001 & $\begin{array}{l}\text { Maestría en Investigación Social Interdisciplinaria, Universidad Distrital } \\
\text { Francisco José de Caldas } \\
\text { Maestrante en Educación de Carácter Investigativo, Universidad } \\
\text { Tecnológica de Bolívar. }\end{array}$ \\
\hline MG002 & Maestría en Desarrollo Infantil, Universidad de Manizales \\
\hline GC003 & Maestría en Educación, Universidad Santo Tomás de Colombia \\
\hline DR004 & Magister en Lingüística, Universidad Nacional de Colombia \\
\hline OV005 & Maestría en Educación, Universidad del Norte \\
\hline \multirow[t]{2}{*}{ MC006 } & $\begin{array}{l}\text { Master en Investigación Documental (Recherche Fle), Université de } \\
\text { Besancon (Francia) }\end{array}$ \\
\hline & Especialista en Investigación Cualitativa \\
\hline CL007 & $\begin{array}{l}\text { Doctor en Educación, Universidad Complutense de Madrid } \\
\text { Maestría en Filosofía y Ciencias de la Educación (España) }\end{array}$ \\
\hline
\end{tabular}

Se identificaron concepciones y creencias de los profesores de acuerdo con las categorías conceptuales mostradas en el Gráfico 2.

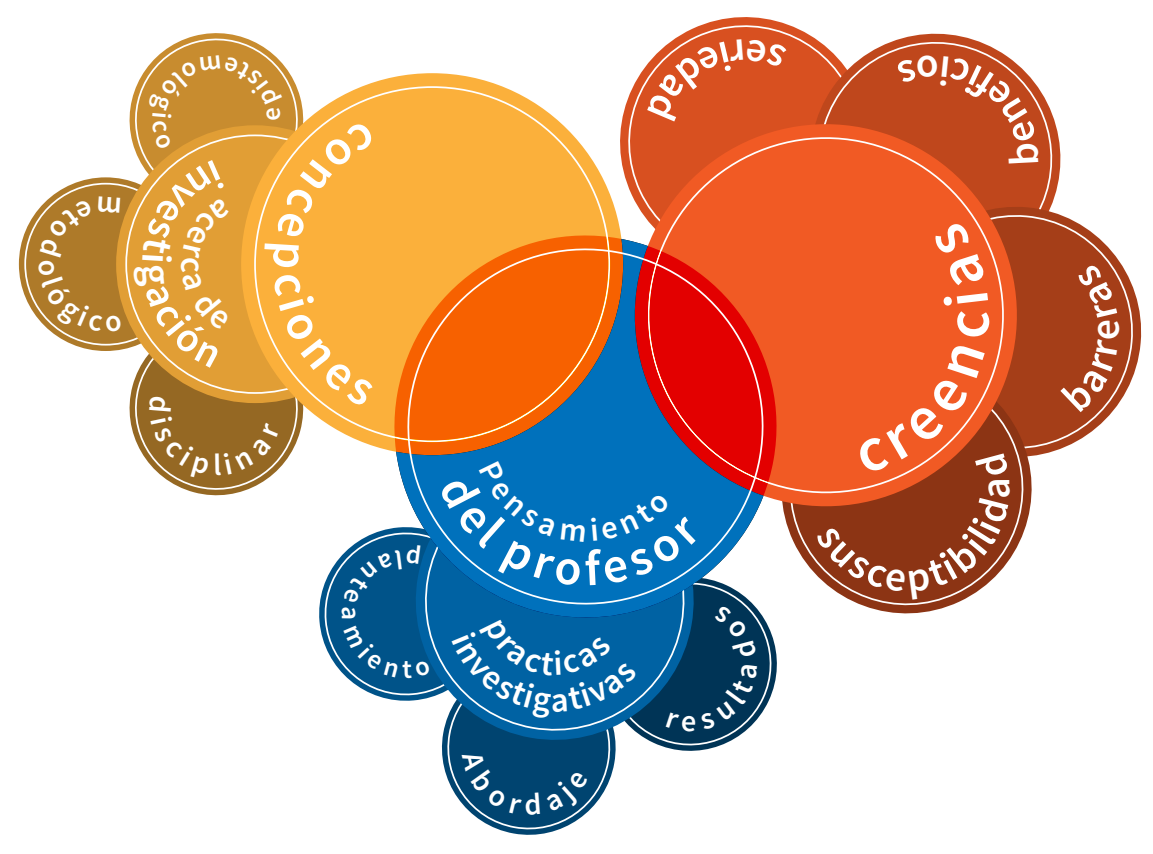

Gráfico 2 Categorías conceptuales

Fuente: elaboración propia

Los textos verbales rescatados de las entrevistas, en cuanto a la categoría concepciones sobre investigación, destacan los siguientes: OV 82-86: "Proceso perceptible que explica la realidad con base a supuestos teóricos así asumo yo la investigación", AS 4-7: "yo concibo la investigación como un indagar, un conocer, un buscar cuales son las causas de x situación que se está presentando y tratar de entenderla para que pueda ver una posibilidad", "bueno la investigación es un ejercicio de búsqueda de aprendizaje para tener claridad sobre nuestro quehacer”, DR 11-13: “... he reconocido que la investigación no es unilateral, existen diversos procesos de investigación para entender los fenómenos”, DR 22-23: "la investigación sistemática es un tipo de investigación desde un enfoque más metafórico", "Casi toda nuestra formación está bajo un paradigma positivista”, MC 12-13: "Mi proceso de formación está dentro de la línea de la hermenéutica", "Participar en eventos y talleres sobre diseño de investigación, metodología.", GC: 45 "Mi formación en investigación se da a partir de cursar la maestría".

En la categoría creencias, se rescataron textos, como: GC 34:41: "la investigación es algo delicado", "es para los inteligentes", "los van a evaluar mal", "van a demostrar que no tienen habilidades", AS 35-39: "La actitud de algunos estudiantes apática hacia la investigación”, KC 34-47: "Hay un aspecto que no, de pronto no manejo mucho porque no manejo ese tipo de investigación, cuando son investigaciones mixtas las investigaciones cuando son de tipo focales, porque no son de mi corriente", "doy más respuestas acertadas con la investigación cualitativa, descriptiva con la investigación acción participación, con la investigación acción, con la etnográfica aquí soy más acertada 
con la respuesta porque es lo que más manejo y con lo que más me gusta trabajar”, CL 29-35: “... investigación permite crecer académicamente...", "...la investigación ayuda a poder actuar en tu entorno de la manera adecuada...", "...las decisiones pedagógicas que tomas posterior a una investigación son más acertados...”, entre muchos.

\section{Discusión}

Producto del ejercicio hermenéutico en la fase del análisis de discurso, emergieron las siguientes categorías:

$\rightarrow$ La Ontológica, aflora su naturaleza humana de querer conocer, el hombre como: ser cognoscente. (Saldarriaga Vélez, 2003)

$\rightarrow$ Yo-Imagen, este componente se presenta al lector, desde la perspectiva lacaniana, el "estadio del espejo". (Lacan, 1949a; 1949b)

$\rightarrow$ La metacognición se visibilizó cuando los profesores investigadores planificaron admitieron compartían con sus estudiantes, el proceso de toma conciencia de lo que está haciendo, cómo lo está haciendo, para qué lo está haciendo, reflexionando sobre cada fase de sus prácticas.

$\rightarrow$ Construcción de conocimiento: "un cambio de orilla, del constructivismo al construccionismo", se reconoció en las coincidencias de los discursos al construir un conocimiento y al promover su construcción, el maestro investigador es consciente de su construcción por propia e interna, integrada en su realidad, supera el enfoque constructivista en el construccionista.

$\rightarrow$ La inclusión, la atención a lo diverso, como elemento que permite un cambio de cosmología pedagógica.

$\rightarrow$ La tradición cultural, la cultura y la tradición son aspectos que revelan tanto, concepciones iniciales como creencias, cumplen con un doble papel, dificultan acoger nuevas teorías para ver la realidad desde otras perspectivas, en este caso sobre la investigación, y, modelar alternativas para establezcan otras formas de pensar y de actuar, esto, dependerá del pensamiento del profesor.

$\rightarrow$ La potencialidad afectiva, relacionada en este estudio con las manifestaciones emocionales frente a la investigación, las preferencias explícitas en los discursos de los profesores, satisfacciones estructurales reveladas en afectos, emociones, sentimientos, etc., o perceptuales, aquellas satisfacciones esperadas. (Pérez López, 1991)

\section{Conclusiones}

Reconociendo que tanto las concepciones como las creencias son construcciones de la mente humana, son barreras o límites perceptibles, invitando a la discusión sobre ello, de allí que todas las otras elaboraciones del pensamiento se vean influenciadas por ambas, se concluye, en algunas, lo siguiente:

\section{De las concepciones y creencias:}

1. Los docentes investigadores participantes en este estudio, conciben a la investigación como un proceso que implica interdisciplinariedad, aunque el tema a investigar corresponda a una disciplina en particular y por medio de ella se realicen acciones que logren observar, describir, explicar y predecir el comportamiento de los fenómenos, pero cuando todos los fenómenos se interconectan, dando origen a fenómenos más amplios, se presenta así la interdisciplinariedad.
2. Según los docentes universitarios consideran que el "paradigma cualitativo", les ofrece las posibilidades para acercarse a problemas de investigación y darles solución; sin embargo, no descartan la posibilidad de apoyarse en el "cuantitativo". Está consideración la asumen, porque este paradigma les permite, indagar, conocer, buscar, describir, aplicar estrategias que le posibiliten construir el nuevo conocimiento.

3. En cuanto a cómo conciben el problema de investigación, los docentes investigadores consideraron que es muy importante contextualizarlo, interpretarlo, mediante de reflexión y análisis de la realidad para luego buscar caminos y soluciones.

4. En el mismo sentido consideran que los procesos hermenéuticos, les permite, comprender e interpretar, para construir y reconstrucción lo observado en la problemática, para plantearla y describirla con fidelidad.

5. La concepción epistemológica de la investigación, de los profesores investigadores de las IES de Cartagena, participantes en este estudio, está circunscrita a las bases teóricas sobre las cuales, razonan, entienden e interpretan la realidad para proponer soluciones a necesidades y problemas de investigación.

6. Los docentes universitarios consideran que, aunque el tema a investigar corresponda a una disciplina en particulary por medio de ella se realicen acciones que logren observar, describir, explicar y predecir el comportamiento de los fenómenos, la investigación, implica la interdisciplinariedad.

7. También se reconoce que todavía algunos investigadores perciben la investigación como un proceso difícil y exigente.

8. Se evidencia que en cada espacio que comparte el profesor universitario con los estudiantes, en su discurso propone, de manera consciente o inconsciente, un abanico de ideas para posibles investigaciones; estas, estarán vinculadas tanto a teorías, como a creencias del profesor.

9. El maestro investigador participante es este estudio, muestra en sus concepciones, un alto nivel de compromiso con la investigación, él es quien debe guiar con disciplina y orientar la realización del trabajo investigativo; en este quehacer impregna el proceso de su conocimiento, ideas y sentimientos, aportando singularidades tanto a la planeación y estructuración del proceso como a su orientación y conclusión.

10. Las concepciones recabadas indican que para los profesores investigadores la investigación es el vehículo para que de forma organizada se logre la transformación de pensamiento y la generación permanente de conocimientos.

\section{Relación entre formación y prácticas pedagógicas investigativas:}

1. Todo proceso de investigación debe partir de un contexto que le permita al docente elaborar un diagnóstico de aquellos problemas que se pueden presentar en contextos específicos. Este contexto permite recopilar la mayor información posible y hay partir de ahí comenzar a realizar todo el proceso para poder darle una posible respuesta a ese problema. Es así como se denota de una manera clara que para iniciar una investigación se debe contextualizar primero la problemática para luego darle paso a la producción de la investigación.

2. El docente investigador, en un intento, orienta el aprendizaje sobre investigación en las prácticas hacia la construcción de su propio saber pedagógico, competencia que según Foucault (1969) debe aprovecharse de nuevos saberes para construir un nuevo discurso. 
3. Los profesores investigadores participantes en este estudio están en capacidad para guiar y orientar con disciplina la realización del trabajo investigativo, porque se han formado académicamente en el área, esto garantiza sus posturas epistemológicas.

4. Los participantes en sus entrevistas mostraron características, identidades y diferencias tanto en comportamientos como en abordajes del proceso investigativo, dado en cada caso, por su nivel de formación, y experiencia profesional, fueron revelando como han ido adquiriendo un estilo propio, como fueron se reacomodando y ajustado a las nuevas exigencias tanto de la investigación como del grupo de estudiantes y de sus formas de trabajo particulares.

5. Los profesores a partir de su formación como profesionales, ven sus creencias y concepciones expuestas a la teoría, esto origina cambios en su pensamiento, aunado esto a la confrontación después en la práctica, se exponen a nuevos cambios de orden metodológico originados por el tipo de investigación. Esto, induce a que sea reflexivo y este abierto a nuevos métodos investigativos, para poder entender y solucionar la realidad del problema e, influyen tanto en lo profesional como en lo personal.

6. En el entorno de las prácticas pedagógicas investigativas se generan lazos afectivos incidentes en la relación profesor estudiante y al mismo tiempo, son un factor determinante para la calidad del proceso de investigación.

7. El ideal, sería vincular tempranamente a los estudiantes a los procesos de investigación, tener en cuenta sus preferencias, frente a estas, reaccionar conjuntamente, profesor y estudiante, para establecer plan de acción mancomunado, frente a las diversas actividades de la investigación.

8. Los profesores investigadores a través de sus experiencias han descubierto que los componentes de una investigación pueden originarse y encontrarse en el contexto de su propia experiencia investigativa y no tienen que provenir necesariamente de los planteamientos sacados de teorías, adquiriendo entonces mayor relevancia social y científica, en cuanto a conocimiento y a uso y apropiación de la misma.

9. La práctica investigativa además de beneficiar con nuevos saberes y la reconstrucción de ellos también mejora las relaciones interpersonales entre estudiante y docente ya que creen y ratifican que la investigación le permite al sujeto crecer académicamente buscar más de lo que él sabe, todas estas creencias muchas veces tienen o pueden tener implicaciones importantes en el camino y desarrollo de su práctica educativa y en la creación de su nuevos conocimiento profesionales para así poder estar abierto al cambio a nuevas teorías y procesos investigativas, como también los afirma Arias Castilla (2014) en su escrito sobre aportes a la calidad en Educación Superior y la evaluación de prácticas en la formación pedagógica.

Las concepciones sobre investigación son propias del investigador, cada uno interpreta, investiga y transforma a partir de lo que ha observado, concibiendo el fenómeno de forma propia. Las concepciones de los docentes sobre investigación parten de problemas evidenciados en los contextos de enseñanza y aprendizaje, allí, reconocen y toman conciencia del problema o del fenómeno de interés para estudiar, actúan reactivamente a través de la creación, e innovación y capacidad investigativa, y son proactivos cuando proponen más allá de lo observado; todo depende de las construcciones propias del sujeto ya que es el quien construye y reconstruye su conocimiento partiendo de las experiencias que tenga del medio donde se desenvuelve.

\section{De las creencias.}

1. El valor que el docente conceda a una creencia va a influir en la inter relación entre estudiante-docente e investigación (susceptibilidad percibida), por ser el área más exigente, desde la perspectiva mutua, donde el docente debe ajustarse y dominar parámetros estrictos (rigor- seriedad percibida) para lograr los resultados esperados.

2. El estudiante y el profesor investigador deben superar las concepciones espontáneas y creencias sobre el proceso de investigación, generando barreras y susceptibilidades de parte y parte. Pueden verse reveladas en inflexibilidad, en no permitirle al estudiante mostrar sus habilidades, conocimientos, o generando miedos frente a la investigación.

3. Los profesores investigadores deben buscar estrategias que permitan a los estudiantes superar creencias relacionadas con "que no son buenos para esto la investigación", que es para los inteligentes etc., el docente debe reflexionar e inducir procesos Meta cognitivos, referidos a como el estudiante aprende a investigar, haciéndolo consciente de los cambios paulatinos en sus propios procesos de pensamiento, del desarrollo de sus propias habilidades (seriedad percibida), esto le permitirá abordar la teoría desde una perspectiva más profesional.

4. La formación de un sujeto como docente esta determina desde un comienzo por lo que quiere es decir determinada por sus deseos y creencias de que es bueno o no en un área específica de la educación (susceptibilidad percibida), es ahí donde empieza su formación ya que esta se va a ver fundamentada por sus experiencias personales, sociales y educativas que lo van a llevar a ser todo un profesional en su campo (beneficios percibidos).

5. La investigación va más allá de los límites de un individuo investigador para ubicarse en la realidad de los problemas, Esto constituye una visión transindividual de los procesos de investigación (seriedad percibida). Cuando un investigador cualquiera elige un tema de estudio y se formula una pregunta y unos objetivos de trabajo, en realidad lo que hace es inscribirse dentro de una problemática en la que también trabajan y han estado trabajando otros investigadores, que suele tener en el tiempo toda una trayectoria de desarrollo y que, a su vez, mantiene sucesivas y complejas conexiones con otras investigaciones, ya que retoman planteamientos previamente formulados y se basan en intentos y soluciones anteriores a ellos, sin tener que comenzar desde cero(barreras percibidas)

6. En el procesos de formación de profesores es menester garantizar la calidad en la enseñanza de nuevos procesos investigativos, habilidades e intereses sociales en sus estudiantes para que adquieran las competencias investigativas que les permitan abordar los problemas de su entorno, reconociendo las necesidades, abordar la investigación desde un lugar epistemológico, para ser innovadores y críticos en estos procesos, fomentando al unísono, el espíritu científico tanto del estudiante como de sí mismo. (beneficios percibidos)

7. Tanto los diseños como los métodos para investigar varían en su aplicación al depender del problema de investigación, esto es percibido como una barrera, si el investigador tiene métodos predilectos al momento de investigar; se recomienda a los docentes darse la oportunidad de innovar metodológicamente (susceptibilidad percibida).

8. La práctica investigativa de cada docente universitario en general, van influyendo en su entorno académico fundamentalmente en la generación del pensamiento, opiniones, aptitudes y actitudes (beneficios percibidos) Igualmente en su formación individual y pedagógica y todo lo que ello conlleva se encuentra determinada principalmente, por 
la cultura que lo rodea y que, en cierta manera, van a determinar esa generación de pensamientos, actitudes. (barreras percibidas).

9. las creencias influyen en la organización y en el uso del tiempo que hacen los profesores; por lo que el factor 'tiempo' es un elemento determinante de los demás aspectos que influyen en la construcción de las creencias de los maestros nos damos cuenta que muchos piensan y tienen la creencia de que la investigación es solo para personas dedicadas al campo investigativo y no buscan estrategias donde muestren la investigación como algo agradable y que se puede desarrollar a través de herramientas diferentes.

10. Las creencias difieren notablemente del conocimiento, pues son algo más subjetivo y afectivo, algo más personal que el conocimiento, además de que son más discutibles, son una parte fundamental del conocimiento que poseen los docentes.

Por último, se recomienda a quienes les interese este trabajo ahondar en las categorías emergente, para ello se dejan a disposición en los anexos las entrevistas.

\section{Trabajos citados}

Álvarez Álvarez, C., \& San Fabián Maroto, J. L. (2012). La Elección del Estudio de Caso en Investigación Educativa. Gazeta de Antropología, 28(1), 14. Obtenido de http://www.ugr.es/ pwlac/G28 14Carmen Alvarez-JoseLuis SanFabian.html

Apple, M. W. (1979). Ideología y Currículo [Ideology and Curriculum] (illustrated reprint translated [es 1ed:1986 2eed:2008] ed.). (R. Lassaleta, Trad.) New York [us]: Routledge \& Kegan Paul [1979] Akal SA [1986,2008].

Arias Castilla, C. A. (2014). Aportes a la Calidad en Educación Superior: Evaluación de prácticas en la formación pedagógica. Trilogía: Ciencia, Tecnología y Sociedad(10), 119-144. Obtenido de http://itmojs.itm.edu.co/index.php/ trilogia/article/view/594

Ayala Rengifo, M. L., \& Martín Cardinal, M. C. (2010). Creencias sobre Lúdica de un Grupo de Docentes que Trabajan en el Nivel de Preescolar en la Ciudad de Bogotá. II Congreso Internacional VII Seminario Nacional. Investigación en Educación, Pedagogía y Formación Docente. Bogotá D.C. [co]:

Bachelard, G. (1938). La formación del espíritu científico: contribución a un psicoanálisis del conocimiento objetivo [La Formation de l'esprit scientifique: contribution à une psychanalyse de la connaissance objective] (illustrated reprint translated [es 1ed:1948 25ed:2004] ed.). Dijon [fr]: Libraire Philosophique [fr 1938]; Argos [ar 1948]; Planeta [es: 1985]; Siglo XXI [mX 2004].

Becker, M. H., \& Maiman, L. A. (1975). Sociobehavioral Determinants of Compliance with Health and Medical Care Recommendations. Medical Care, 13(1), 10 24. doi:10.1097/00005650-197501000-00002

Beuchot, M. (2004). Hermenéutica, Analogía y Símbolo (191 ed.). México D.F. [mx]: Herder.

Brody, C. M., \& Day, M. (1993). Co-Teaching, Teacher Beliefs and Change: A case study. AERA Annual meeting: The Art and Science of Educational Research and Practice,. Atlanta [us]: AERA, The American Educational Research Association.

Bromme, R. (1988). Conocimientos Profesionales de los Profesores [Institut Für Didaktik Der Mathematik Universität Bielefeld]. (G. Guijarro Puebla, Trad.) Enseñanza de las Ciencias, 6(1), 19-29. Obtenido de https://ddd.uab.cat// pub/edlc/02124521v6n1/02124521v6n1p19.pdf

Cicourel, A. V. (1980). Three Models of Discourse Analysis: The role of social structure. Discourse Processes, 3(2), 101-131. doi:10.1080/01638538009544482

Clarke, D., \& Hollingsworth, H. (2002). Elaborating a Model of Teacher Professional Growth. Teaching and Teacher Education, 18(8), 947-966. doi:10.1016/ S0742-051X(02)00053-7
Claxton, G. (1997). Cerebro de liebre, mente de tortuga: por qué aumenta nuestra inteligencia cuando pensamos menos [Hare Brain, Tortoise Mind: How Intelligence Increases When You Think Less] (illustrated reprint translated [es 1ed:1998 4ed:2016] ed.). London [uk]: Fourth Estate; Harper Collins [2016].

Davies, P. (1993). La Mente de Dios: la base científica para un mundo racional (264 ed.). Madrid [es]: McGraw-Hill.

Foucault, M. (1969). La Arqueología del Saber (355 [79] ed.). (A. Garzón del Camino, Trad.) México D.F. [mx]: Siglo XXI.

Habermas, J. (1967). La Lógica de las Ciencias Sociales (506 ed.). Madrid [es]: Tecnos.

King, K., Shumow, L., \& Lietz, S. (2001). Science Education in an Urban Elementary Schools: Case studies of teacher beliefs and classroom practices. Science Education, 85(2), 89-110. doi:10.1002/1098-237X(200103)85:2\%3C89::AIDSCE10\%3E3.0.CO;2-H

Lacan, J. (1949a). Le Stade Du Miroir Comme Formeteur de la Fonction Du Je: Tellequ'elleno usestrévé lé edans l'expérience psychanalytique. XVIe Congrès international de psychanalyse, à Zurich le 17-07-1949. 13, págs. 449-455. París [fr]: Association Psychoanalitique Internationale. Obtenido de http://gallica.bnf.fr/ark:/12148/bpt6k54444473/f3.image

Lacan, J. (1949b). El Estadio del Espejo. En J. Lacan, J. Lacan, J. D. Nasio, \& A. Suárez (Edits.), Escritos I (págs. 99-105). Buenos Aires [ar]: Siglo XXI. Obtenido de https://www.epbcn.com/pdf/jose-maria-blasco/1992-10-22-Elestadio-del-espejo-Introduccion-a-la-teoria-del-yo-en-Lacan.pdf

Merriam, S. B. (1997). Qualitative Research and Case Study Applications in Education: Revised and expanded from case study research in education (undefined ed.). San Francisco [us]: Jossey-Bass.

Moreno Moreno, M., \& Azcárate Giménez, C. (2003). Concepciones y Creencias de los Profesores Universitarios de Matemáticas acerca de la Enseñanza de las Ecuaciones Diferenciales. Enseñanza de las Ciencias, 21(2), 265280. Obtenido de http://www.raco.cat/index.php/Ensenanza/article/ download/21935\%20/21769

Morin, E. N. (1986). Introducción al pensamiento complejo (iLLustrated reprint translated [es 1ed:1990 2ed:1998] ed.). (M. Pakman, Ed., \& M. Pakman, Trad.) Valladolid [es]: Universidad de Valladolid; Gedisa [1990].

Patton, M. Q. (1990). Qualitative Evaluation and Research Methods (169-192 [598] ed.). London [uk]: SAGE Publications.

Perafán Echeverri, G. A. (2002). Pensamiento y Conocimiento de los Profesores: Debates y perspectivas internacionales. Bogotá D.C. [co]: UPN, Universidad Pedagógica Nacional.

Pérez López, J. A. (1991). Teoría de la Acción Humana en las Organizaciones: La acción personal ([209] 304 ed.). Madrid [es]: Riapl.

Porlan, R., Rivero García, A., \& Martín del Pozo, R. (1998). Conocimiento Profesional y Epistemología de los Profesores II: Estudios empíricos y conclusiones. Enseñanza de las Ciencias, 16(2), 271-288. Obtenido de http://www.raco. cat/index.php/Ensenanza/article/view/21534

Pozo, J. I. (2006). Educar para la Sociedad del Conocimiento: La reconstrucción mutua de mente y cultura. IV Congreso Internacional de Educación. Buenos Aires [ar]: [unreachable].

Quintero Mejía, M., \& Ruíz Silva, A. (2004). ¿Qué Significa Investigar en Educación? Bogotá D.C. [co]: Universidad Distrital Francisco José de Caldas.

Saldarriaga Vélez, O. d. (2003). Del Oficio del Maestro: Prácticas y teorías de la pedagogía moderna en Colombia (320 ed., Vol. 2). Bogotá D.C. [co]: Cooperativa Editorial Magisterio.

Schön, D. A. (1987). Educating the Reflective Practitioner (355 ed., Vols. JosseyBass Higher Education Series). San Francisco [us]: Willey.

van Dijk, T. A. (2001). Algunos Principios de una Teoría del Contexto. Revista Latinoamericana de Estudios del Discurso, 1(1), 69-81. Obtenido de http://discursos.org/oldarticles/Algunos\%20principios\%20de\%20una\%20 teor\%EDa\%20del\%20contexto.pdf

Vygotsky, L. S. (1978). Interaction Between Learning and Development. En M. Gauvian, \& M. Cole (Edits.), Readings on the development of children (34-41 [384] ed., págs. 34-41 [384]). New York [us]: Worth Publishers.

Yin, R. K. (1994). Case Study Research: Desing and methods (undefined ed., Vol. 5 Applied social research methods series). London [uk]: SAGE Publications. 